\section{LA INTEGRACIÓN URBANA: UNA META DESEADA, PERO SIN DIAGNÓSTICO NI PROPUESTA DE SOLUCIÓN INTEGRAL}

\author{
Margarita Greene $e^{1}$ y Camilo Arriagada ${ }^{2}$
}

La convocatoria de este número especial estaba enfocada en la integración urbana y su contracara: la exclusión. La meta de la integración y el rechazo a la exclusión, han compuesto una imagen objetivo declarada y consensuada respecto al tipo de desarrollo deseado para las ciudades latinoamericanas, que fue formalizada en la agenda internacional de Hábitat y en nuestra Política Nacional de Desarrollo Urbano.

Abunda información que describe los efectos beneficiosos de la integración y, mucho más, sobre las consecuencias perniciosas de la exclusión. Sin embargo, la definición de integración, su medición y discusión (en términos de qué causa la exclusión o

1 Chile. Investigadora Principal CEDEUS. Profesora titular, Escuela de Arquitectura Pontificia Universidad Católica de Chile, https://orcid.org/0000-0001-9105-0502. Correo electrónico: mgreenez@uc.cl

\section{URBAN INTEGRATION: A DESIRED GOAL, BUT WITHOUT DIAGNOSIS OR COMPREHENSIVE SOLUTION PROPOSAL}

de cuáles serían medidas efectivas para contrarrestarla: como políticas públicas, rol de agentes privados o mercado y espacios formales para la ciudadanía), son procesos aún en desarrollo o construcción. Conscientes de que, por un lado, se trata de conceptos polisémicos, multi-dimensionales y con características multi-escalares y que, por otro lado, las propuestas de políticas públicas que invocan su nombre son demasiado amplias o insuficientes, quisimos invitar a una discusión más acabada.

En efecto, aún persisten ambigüedades o indefiniciones respecto al concepto de integración urbana, en cuanto a su expresión concreta o atributos, y también recurrentes vinculaciones con temas de

2 Chile. Profesor asociado Facultad de Arquitectura y Urbanismo, Universidad de Chile. Correo electrónico: carriagadal@uchilefau.cl 
cohesión y equidad (o sus antípodas, exclusión, inequidad y fragmentación urbana). Sabemos que la integración urbana es un atributo requerido para el desarrollo de ciudades sustentables, y es también una demanda importante al considerar potenciales reformas de políticas urbanas a escalas central, regional y local. Hoy también ha surgido con fuerza un debate sobre su nexo con la instalación de estructuras e instrumentos de gobierno metropolitano.

Como resultado de la convocatoria, recibimos 63 artículos, que entregaban una amplia gama desde la cual seleccionar. Para los artículos de este número buscamos, primero, marcos conceptuales alternativos para analizar una casuística de territorios o piezas urbanas que reflejaran viejas y nuevas expresiones de la desigualdad estructural de la sociedad y que, acumuladamente, armaran una suerte de tipología de situaciones que deterioran o parcializan la integración de personas, hogares y barrios.

Así, el primer artículo "Integración urbana y calidad de vida: Disyuntivas en contextos metropolitanos", de Vicuña, Orellana, Trufello y Moreno, explora en qué medida la integración urbana se relaciona con una mejor calidad de vida en las áreas metropolitanas de Santiago, Valparaíso y Concepción. Mediante el análisis comparado del Índice de Calidad de Vida Urbana (ICVU) e índices de Integración Urbana levantados a partir del Sistema de Indicadores y Estándares de Desarrollo Urbano (SIEDU), abordan tres dimensiones clave: condiciones socioculturales, conectividad y movilidad, y vivienda y entorno. Su análisis muestra que la integración urbana no necesariamente se expresa como resultado de una mejor calidad de vida, y abre un importante cuestionamiento sobre los alcances que puede tener una buena dotación de bienes y servicios públicos y privados para garantizar un mayor grado de integración en barrios y comunas, al menos a escala metropolitana. Adicionalmente, se devela la importancia que reviste la geografía de las áreas metropolitanas, debido a la obtención de algunos resultados contrapuestos, generando un aporte significativo al diseño de políticas públicas.

Luego de este primer artículo, que marca la pauta de este número, se reconocen dos líneas de desarrollo ancladas en la marginalidad, característica propia del fenómeno de los asentamientos informales ("slums"). Si bien estos no son algo nuevo en la realidad latinoamericana, siguen existiendo bien entrados al siglo XXI, pero en otro contexto y modelo de desarrollo. Así, aún cuando siguen proyectando la marginalidad dura, sus magnitudes son menores y tienen una lógica de enclave nueva. Ya no se trata del fenómeno masivo, propio de las fases álgidas de migraciones campo-ciudad de inicios del siglo XX, y que llevaron a fundar la sociología urbana alrededor de los paradigmas de marginalidad y necesidades básicas insatisfechas. 
En la primera línea hay dos artículos: el primero, "La integración social como desafí: El Programa de Campamentos en Chile (2011-2018)", de Matus, Ramoneda y Valenzuela, describe estos asentamientos como una política de acceso al suelo, saneamiento e infraestructura de la ciudad formal. Hoy, cuando se pensaba que era un fenómeno erradicado del paisaje urbano, vuelve a rebrotar en la ciudad globalizada donde la accesibilidad y conectividad a fuentes de ingreso se vuelven tan fundamentales que muchas personas prefieren residir en micro campamentos incrustados en vacíos e intersticios urbanos, a excluirse de los beneficios que ofrece la ciudad. Se analizan los resultados de catastros de campamentos y se realiza un estudio de cuatro casos de estrategias de cierre de estos en diferentes regiones del país. El artículo releva la importancia de incorporar dimensiones como el afecto y apego, el sentido de pertenencia y la participación, en la generación de integración social; ello permite discutir algunas perspectivas sobre la integración basadas exclusivamente en la cercanía espacial.

El siguiente artículo se concentra en el problema de los guetos, vecindarios populares atravesados por la inseguridad y el tráfico de drogas: "Configuración territorial del hábitat en un asentamiento informal en Montería, Colombia", de Vega, Hernández y Barbera. Es un artículo simbólico, porque ilustra los efectos de las fracturas de integración y normatividad que, exacerbados por la economía y sociedad de consumo desigual globalizada, afectan la esencia de la ciudad como artefacto histórico de encuentro, progreso y bienestar público y privado. Se concluye que el asentamiento es una mezcla humana, un escenario en el cual los actos de habitar construyen su territorio con la intención de integrarse e interrelacionarse con los sectores circundantes, y desdibujar el fenómeno de segregación social y fragmentación del hábitat. Por ello, el control territorial debe estar dado por los procesos de organización y liderazgo local, como una estrategia para lograr condiciones urbanas de habitabilidad. No obstante, la gran producción y diversificación de estudios de segregación urbana regional y mundial, destacan que el problema no se resolverá con proyectos aislados de integración, sino con una nueva generación de políticas de desarrollo que llene el vacío que dejó el tránsito vertiginoso hacia la economía de consumo global, con la respectiva desvalorización del trabajo y la educación de las mayorías.

La segunda línea avanza en el tema de los asentamientos informales. En "Estrategias de integración urbana en contextos de informalidad y avance de la inseguridad", de Gargantini, se indaga sobre las relaciones sinérgicas entre segregación, seguridad, narcotráfico, espacio público y participación ciudadana (desde los postulados del urbanismo social y del urbanismo táctico en pos del mejoramiento y revalorización del espacio público), a partir de la caracterización y análisis crítico del modelo de intervención integral en Córdoba, Argentina. Se exponen herramientas de diseño y gestión urbana 
factibles de ser implementadas en barrios de esta naturaleza, ofreciendo un modelo de intervención. Su aplicación da cuenta que, frente a la pérdida de valor de los espacios educativos y productivos, las áreas deportivas y para el ocio resultan efectivas para lograr integración en nuestras urbes. Esto supone una valorización de variables culturales y la participación de la ciudadanía en solucionar los problemas, para potenciar el desarrollo de mejores políticas integradoras.

También desde esta segunda línea, en "Segregación residencial socioeconómica e inserción laboral: El caso de San Carlos de Bariloche, Argentina", Niembro, Guevara y Cavanagh sostienen que la segregación residencial socioeconómica puede considerarse uno de los principales obstáculos para una adecuada integración urbana, ya que suele entrelazarse con diversas problemáticas sociales contribuyendo a su reproducción inter-generacional. El artículo explora en los vínculos entre la segregación residencial y la inserción laboral de la población mediante una combinación de técnicas de análisis multivariado y datos del último censo poblacional (2010); se obtiene, primero, una tipología que permite diferenciar a los barrios de la ciudad y, luego, se analiza la integración al mercado de trabajo de la población residente en ellos. Los resultados están en línea con trabajos previos realizados en Latinoamérica, que muestran que quienes habitan en entornos segregados suelen tener mayores dificultades de inserción laboral y, con ello, de integración y progreso social. Por otro lado, parte de la evidencia no sólo parece contradecir las tradicionales tesis de desajuste espacial o efecto vecindario, sino también mostrar algunos límites de las políticas públicas para incidir en la integración urbana plena, si sólo se limitan al acceso a la vivienda y servicios básicos.

Un estudio que aborda el tema de la segregación y exclusión desde una perspectiva más particular, pero también relevante en muchas de nuestras ciudades, es "Turismo, gentrificación y presión por desplazamiento en Valparaíso, Chile", de Cáceres. Aquí se discute la renovación urbana en zonas patrimoniales (cerros Alegre y Concepción) impulsada por la industria turística-inmobiliaria, y promovida por el Estado mediante programas y subsidios. Se busca comprender cómo el habitante experimenta y significa estos cambios. Los resultados muestran un turismo que ha valorizado viviendas y mejorado el equipamiento urbano, al mismo tiempo que ha generado una pérdida de vida de barrio y aumentado la vulnerabilidad de los residentes. Así, el caso refleja bien los claro-oscuros de la globalización mediante procesos paradojales paralelos: por un lado, de patrimonialización, mercantilización de lo tradicional y cultural, a través del desarrollo de circuitos turísticos con rasgos gentrificadores, y por otro, en un cuadro de aguda fragmentación urbana y desplazamiento, por encarecimiento del suelo y la vivienda junto a las tensiones propias de la transformación de la identidad barrial y urbana. 
El último artículo, "La lucha por la vivienda en España (2009-2019): desde la calle a las instituciones", de Álvarez y Smith, presenta un estudio sobre la lógica del urbanismo financiero y sus efectos en la asequibilidad a la vivienda, generando una nueva generación de movimientos de demanda y reivindicación. Se analiza el proceso español de la última década (2009-2019), de "lucha" por hacer efectivo el derecho la vivienda, desde la "toma de la calle" a la "toma de las instituciones", basándose en la teoría de la estructuración de Giddens, para valorar la capacidad de estos procesos en transformar las relaciones de autoridad, la asignación de recursos y los sistemas de significado. Este artículo fue colocado al cierre para mostrar el alcance internacional de las transformaciones que genera la globalización y bancarización del fenómeno inmobiliario y su efecto remodelador de las ciudades y los derechos de ciudadanía urbana.

Resumiendo, este número se inicia y termina con artículos que presentan visiones amplias y globales de la integración urbana desde perspectivas muy diferentes. El primero, una operacionalización del concepto y un análisis de su relación con la calidad de vida; mientras que el segundo, desde una perspectiva más disciplinar, explora el significado profundo de quedar excluido del derecho a la vivienda. Entre ambos extremos se presentan cinco casos específicos de interesantes respuestas parciales relacionadas con cómo trabajar la integración urbana, acotada o parcial, y se aporta sobre las variantes de programas o proyectos urgentes para prevenir la existencia de deterioros mayores en ésta. La idea es reflejar el estado del arte, en el tema de la convocatoria, en toda su complejidad.

No obstante, es importante señalar que queda de manifiesto una tendencia metodológica al estudio de casos urbanos únicos, que contrasta con la internacionalización de los capitales, y modelos de desarrollo y comunicaciones, que promueve la nueva economía inmobiliaria, unida al desmantelamiento de la planificación y su nexo con modelos de desarrollo nacionales. Sorprende, asimismo, quizás en relación al creciente imperio de la fragmentación y ausencia de planificación urbana, la desaparición de los códigos estructurales o morfológicos del crecimiento urbano: centros históricos, pericentro, periferias y suburbios, tienden a disolverse como códigos de referencia de la ciudad completa o el barrio. No sabemos si esto es por pragmatismo, o por perplejidad de las investigaciones urbanas.

Para el futuro, quedan de manifiesto las urgencias no resueltas de este número, en el sentido de volcar estudios al nexo de resultados con la instalación de estructuras e instrumentos de gobierno metropolitano, decantar el aporte de la noción de integración urbana, más allá del marketing político, con el vacío de estrategias de gobierno y espacios sustantivos para el fomento de la cohesión, equidad, 
y organicidad del desarrollo urbano, y cuáles estándares de consenso debieran definir la presencia de integración urbana en el logro de mejores políticas que las actuales, ya que su vacío contribuye a explicar algunas de las situaciones y manifestaciones descritas en los estudios presentados.

Santiago de Chile, octubre de 2019. 